\title{
Integrated Focused Ion Beam-Ultramicrotomy Method for TEM Specimen Preparation of Porous Fine-Grained Materials
}

\author{
Kenta K. Ohtaki ${ }^{1}$, John P. Bradley ${ }^{1}$ and Hope A. Ishii ${ }^{1 *}$ \\ 1. University of Hawaii HIGP, Honolulu, USA \\ * Corresponding author: ishii3@hawaii.edu
}

In extraterrestrial sample studies, we need to prepare electron-transparent $(<100 \mathrm{~nm})$ cross-sections for transmission electron microscopy (TEM) from few-centimeter-sized, one-of-a-kind samples that contain large crystals but may also have very fine-grained, porous regions that can contain amorphous grains. Here, we present a new method that integrates focused ion beam (FIB) and ultramicrotomy to overcome the challenges of fine-grained, porous materials in bulk samples. We demonstrate its use on the finegrained matrix of the Paris meteorite that contains amorphous matrix and crystalline inclusions.

A major advantage of FIB sample preparation is that specific locations on samples can be extracted as TEM specimens directly from the bulk. Disadvantages are the large footprint of material removal (typically $\sim 20$ x $40 \mu \mathrm{m}$ for a single FIB section), ion beam damage and re-deposition, and "curtaining". During specimen thinning, incident ions are deposited into the surface and create an amorphized layer with thickness dependent on incident angle, beam energy and sample materials[1,2]. Gradually reducing incident ion beam energy (low-kV polishing) can reduce the ion-induced amorphous layer thickness to $\sim 3-5 \%$ of the total section thickness, but it remains significant for samples with indigenous amorphous components. Re-deposition of milled material is most significant for porous samples; pores can be filled, obscuring the original morphology and creating amorphous regions that can be difficult to distinguish from indigenous amorphous materials without extensive elemental mapping. Moreover, samples with pores and/or multiple phases can result in "curtaining" on the final TEM specimen, fluctuations in sample thickness that complicate data interpretation, due to different milling rates of different phases.

Ultramicrotomy, slicing an epoxy-embedded sample by diamond knife, is well-suited for TEM specimen preparation of porous and/or fine-grained materials; however, only small samples can be prepared[3]. Ultramicrotomy can produce many TEM specimens with nearly-constant thickness from one sample with almost no material loss and free from ion beam damage, re-deposition or curtaining. However, unlike FIB, a survey of the bulk sample surface prior to sectioning is generally not feasible, and if the sample is too large, pull-out of material from the cutting face and/or loss of material from the centers of sections may occur.

For inhomogeneous bulk samples like the Paris meteorite, it is crucial to be able to select sites of interest, and due to its one-of-a-kind nature, the sample size cannot be reduced by grinding to allow ultramicrotomy, despite its suitability for the matrix of this meteorite. To overcome these issues, we developed a new integrated FIB-ultramicrotomy specimen preparation method. Prior sample preparation methods have used FIB to pick up and move single, small grains for ultramicrotomy[4]. Here, a large $\sim 10 \mu \mathrm{m}$ block from a specific site from the bulk Paris meteorite sample was extracted using FIB, and welded to a $\mathrm{Cu}$ grid (Figure 1). The grid was then embedded in low-viscosity epoxy. The $\mathrm{Cu}$ grid is sufficiently soft to allow trimming of a mesa around the block, and TEM specimens were generated by ultramicrotomy. A TEM specimen was also prepared using conventional FIB methods for comparison. 
A much larger quantity of thinner, less-damaged specimens from specific sites in bulk samples are possible with this new integrated FIB-ultramicrotomy method. The footprint for FIB trench milling for the integrated method is approximately the same as for conventional FIB extraction of a single section, but dozens of thin sections were produced with minimal ion beam damage only at sample edges with the integrated method. The microtomed specimens occasionally have a couple of wrinkles, and chattering is observed perpendicular to the sectioning direction (chattering can be reduced by sectioning smaller areas), but over large areas, the thickness is constant $(\sim 80 \mathrm{~nm}$, with thicknesses of $\sim 30 \mathrm{~nm}$ possible in these kinds of materials), and important features are preserved (Figure 2 (a)). The conventional FIB specimen is thicker $(\sim 150 \mathrm{~nm}$, on average, with thicknesses of $\sim 120 \mathrm{~nm}$ possible), and its thickness is highly variable due to sample warping during the thinning process and to pores and cracks that resulted in "curtaining". Thin specimens are critical for fine-grained samples to avoid overlap of constituents in TEM. Finally, the integrated method sections lack the redeposited $\mathrm{Cu}$ seen at the bottom of the conventional FIB specimen.

\section{References:}

[1] JP McCaffrey et al., Ultramicroscopy 87 (2001), p. 97.

[2] J Mayer et al., MRS Bulletin 32 (2007), p. 400.

[3] LG Melo et al., J Power Sources 312 (2016), p. 23.

[4] Y Xu et al., Earth Planets Space 70 (2018), p. 150.

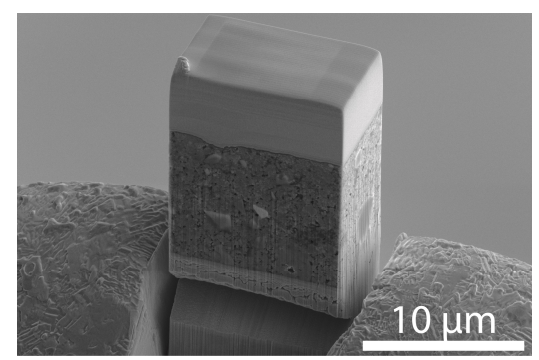

Figure 1. (above right) FIB sectioned block on the center pole of $\mathrm{Cu}$ grid. The bottom is welded with $\mathrm{Pt}$ and the sides are cleaned with ion beam. The sample is now ready for embedding and ultramicrotomy.
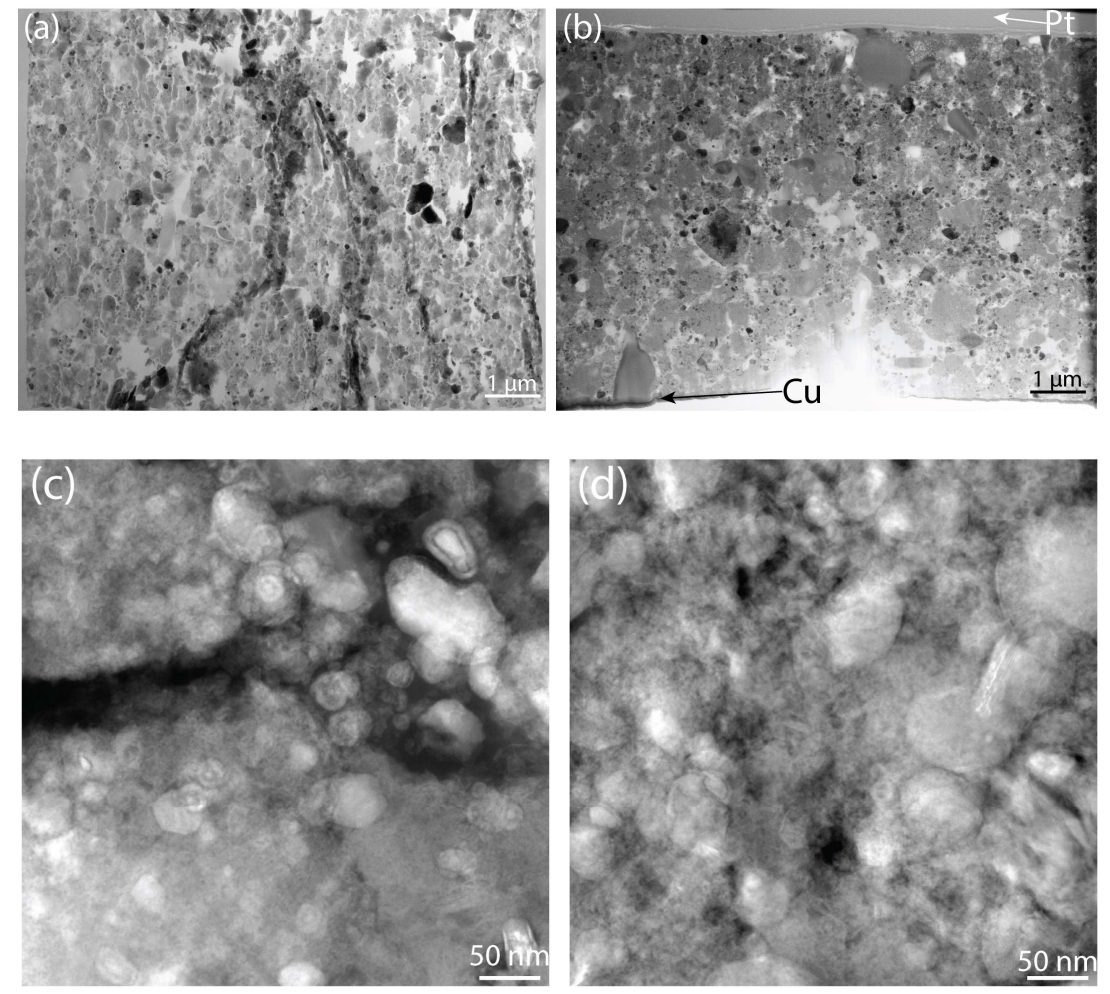

Figure 2. (a) Bright field image of the Paris matrix specimen prepared using the integrated FIBultramicrotomy method. (b) Bright field image of the conventional FIB thinned Paris matrix specimen. (c) High angle angular dark field (HAADF) image of fine features in the integrated FIBultramicrotomy specimen. (d) HAADF image of fine features in the conventional FIB thinned specimen. Due to the thickness with overlapping grains, the FIB thinned specimen lacks sharpness in small features. 